\title{
Electrophysiological evidence during episodic prospection implicates medial prefrontal and bilateral middle temporal gyrus
}

\author{
Chia-Fen $\mathrm{Hsu}_{1,2}$ \& Edmund J.S. Sonuga-Barke ${ }_{1,3}$
}

1 Institute for Disorders of Impulse \& Attention, Developmental Brain-Behaviour Laboratory, Psychology, University of Southampton, UK

2 Department of Psychology, York University, Canada

3 Department of Experimental Clinical \& Health Psychology, Ghent University, Belgium

Corresponding author: Prof. Edmund Sonuga-Barke

Psychology

Shackleton Building (B44)

University of Southampton

Highfield Campus

Southampton SO17 1BJ

UK

Email: ejb3@soton.ac.uk

Phone: +44 (0) 2380594604 
EEG correlates of prospective cognition

\begin{abstract}
fMRI studies have implicated the medial prefrontal cortex and medial temporal lobe, components of the default mode network (DMN), in episodic prospection. This study compared quantitative EEG localized to these DMN regions during prospection and during resting and while waiting for rewards. EEG was recorded in twenty-two adults while they were asked to (i) envision future monetary episodes; (ii) wait for rewards and (iii) rest. Activation sources were localized to core DMN regions. EEG power and phase coherence were compared across conditions. Prospection, compared to resting and waiting, was associated with reduced power in the medial prefrontal gyrus and increased power in the bilateral medial temporal gyrus across frequency bands as well as greater phase synchrony between these regions in the delta band. The current quantitative EEG analysis confirms prior $\mathrm{fMRI}$ research suggesting that medial prefrontal and medial temporal gyrus interactions are central to the capacity for episodic prospection.
\end{abstract}

Key words: Episodic prospection; self-referential thought; default mode; temporal gyrus; Quantitative EEG 
EEG correlates of prospective cognition

\section{Introduction}

The default mode network (DMN) is a set of widely distributed, but functionally connected brain regions including the medial prefrontal cortex (MPFC), posterior cingulate cortex/precuneus (pC) and bilateral middle temporal gyrus (MTG; Laird et al., 2009; Raichle et al., 2001). The DMN is active during wakeful rest. It is also associated with a range of introspective self-referential cognitive states such as episodic prospection (Spreng \& Grady, 2010), autobiographical memory (Addis, Wong, \& Schacter, 2007; Spreng, Mar, \& Kim, 2009), mind wandering (Christoff, Gordon, Smallwood, Smith, \& Schooler, 2009) and moral decision making (Reniers et al., 2012). Although differing in terms of their specific content these mental processes share common features, i.e. they all involve shifting individual's perspective from the present context to an alternative temporal (past or future) or locational (here or there) or social (me or other) context. The DMN plays an important role in the integration of autobiographical information driving decisions relating to desired future states (Sonuga-Barke \& Fairchild, 2012). Central to this is episodic prospection - the ability to mentally 'imagine' or 'preview' possible future events and states (Boyer, 2008; Lin \& Epstein, 2014). Functional magnetic resonance imaging (fMRI) studies have implicated the medial prefrontal cortex and medial temporal lobe as a putative neural network underpinning episodic prospection (Buckner, Andrews-Hanna, \& Schacter, 2008) working with MPFC and MTG to facilitate processes of self-projection into the future (Bar, 2009; Buckner \& Carroll, 2007). This is consistent with the research showing that MTG provides the foundation for internal mentation during autobiographical memory retrieval (Fox, Spreng, Ellamil, Andrews-Hanna, \& Christoff, 2015; Li, Mai, \& Liu, 2014; Tulving, 2002), while 
EEG correlates of prospective cognition

MPFC is implicated in self-related simulations (Kim, 2012; Szpunar, Watson, \& McDermott, 2007) and complex perspective-taking processes (Van Hoeck et al., 2013).

The neural correlates of introspective, self-referential cognition and the resting brain have been investigated previously using a range of different methods such as $\mathrm{FMRI}$ and electroencephalography (EEG) recordings (Christoff, 2012; Helps et al., 2010; Helps, Broyd, James, Karl, \& Sonuga-Barke, 2009; Helps, James, Debener, Karl, \& Sonuga-Barke, 2008; Knyazev, 2013; Knyazev, Savostyanov, Volf, Liou, \& Bocharov, 2012; Smallwood, Brown, Baird, \& Schooler, 2012; Spreng, et al., 2009). Nevertheless, studies of the association between episodic prospection and the DMN have been limited solely to fMRI. Although a considerable amount is known about activations of the DMN regions during episodic prospection, the connectional pattern between core DMN hubs and the roles of different DMN regions in introspective processes remain a matter of debate. Compared to fMRI, EEG provides a direct measure of neural activity with excellent temporal resolution to the millisecond range, and so EEG is well placed to assess the functional coupling of brain oscillations. Previous research has demonstrated a direct connection in resting state functional connectivity between the DMN as identified by fMRI blood-oxygen-level dependent (BOLD) signals and EEG oscillations in very low frequency (Hiltunen et al., 2014), and higher frequency domains (Laufs et al., 2003; Mantini, Perrucci, Gratta, Romani, \& Corbetta, 2007). Strong association between fMRI BOLD signals and EEG oscillations have been observed in anterior MPFC, one of the DMN hubs, during cognitive task performance in both healthy volunteers (Meltzer, Negishi, Mayes, \& Constable, 2007; Scheeringa et al., 2009), and a clinical sample with attention-deficit/hyperactivity disorder (ADHD; Cannon, 
EEG correlates of prospective cognition

Kerson, \& Hampshire, 2011; Cannon, Kerson, Hampshire, \& Garner, 2012). Taken together, EEG offers an ideal platform to investigate brain oscillations during episodic prospection.

In the current research, we extend previous literature to present the first study of episodic prospection using EEG signals localized to the DMN regions. To study prospection we asked participants to consider the use of money in future scenarios. Participants were instructed to imagine themselves actually experiencing and spending money in the scenario compared with a condition when they were asked to estimate the values of items that could be purchased in the scenario (Benoit, Gilbert, \& Burgess, 2011). The imagine condition is designed to involve the recollection of autobiographical or contextual memory, whereas the estimate condition aims to elicit semantic or declarative memory processes. Previous EEGbased studies have demonstrated that EEG correlates can effectively differentiate episodic and semantic memory processes (See Klimesch, 1999 for a detailed review). For example, Klimesch and colleagues measured patterns of event-related desynchronization or synchronization (i.e. the percentage of band power decrease or increase in a test interval as compared to a reference interval), during semantic and episodic memory tasks. In the semantic memory task, participants were asked to judge whether the concept-feature pairs were semantically congruent. In the episodic memory task, which followed the semantic task without warning, participants were asked to report whether specific concept words were actually presented with feature words. Despite the two tasks sharing the same feature words, the extent of alpha power suppression, particularly in the upper alpha band, was related to semantic memory performance, whereas the theta power increase was correlated with encoding new information into episodic memory (Klimesch, Doppelmayr, Pachinger, \& Ripper, 1997; Klimesch, Schimke, \& Schwaiger, 1994). Therefore, we 
EEG correlates of prospective cognition

anticipated there might be differences in EEG activity between the imagine and estimate conditions.

There were two more control conditions of interest: resting and waiting. These allowed the differentiation of specific EEG correlates relating to prospection from those activated during similar brain states. Although both episodic prospection and resting states activate some common neuroanatomical regions within the DMN they are not identical states. For instance, spontaneous thoughts generated during rest are unconstrained and lack a specific objective, whereas prospective thoughts involve definite scenarios and often have a clear temporal focus. In this sense prospection has a more goal-directed and effortful nature than resting. It also involves systematic accessing and integrating memories about prior experiences with thoughts about future possibilities. Given this we expected to see a degree of suppression of core DMN hubs such as the MPFC (seen in goal-directed tasks) and increased activation of MTG (seen in autobiographical memory tasks) during episodic prospection relative to that seen in the pure resting state.

The brain states occurring when individuals are actually waiting for future events also provides an interesting contrast with the process of prospection about those events. Recent EEG studies observed the attenuation of EEG power within the frontal regions when individuals were 'waiting' for rewards compared to when they were resting (Hsu, Broyd, Helps, Benikos, \& Sonuga-Barke, 2013). The waiting-induced attenuation of neural oscillations was localized to midline brain structures overlapping with the DMN regions, suggesting the suppression may be caused by the goal-directed nature of waiting (Hsu, Benikos, \& Sonuga-Barke, 2015). However, there was no evidence of increased MTG activation during waiting, suggesting that once the decision to wait had been taken there 
EEG correlates of prospective cognition

was limited prospective thinking. Moreover, previous research has demonstrated that episodic prospection reduces the discounting of future rewards in healthy volunteers (Benoit, et al., 2011; Peters \& Büchel, 2010), but see negative findings in patients with amnesia (Kwan et al., 2012; Kwan et al., 2015). It is not clear how episodic prospection contributes to the tolerance of delay during waiting. A direct comparison of neural oscillations between prospection and waiting state would provide evidence of the impact of prospective thinking on impulsive decision making.

To increase the power and sensitivity of the study we employed a hypothesis driven, region of interests (ROI) strategy and focused on the signals sourced to the core DMN regions including the bilateral MTGs, MPFC and $\mathrm{pC}$ regions. We predicted that: (i) compared to resting, prospection would be associated with lower activity within the MPFC given its more goal-directed nature; (ii) compared to both resting and waiting conditions prospection would be associated with higher activity in the MTG and higher coherence between MTG and MPFC given its likely use of autobiographical and self-referential elements.(iii) These effects would be most pronounced in the condition where individuals were asked to imagine spending money rather than just estimating the cost of things.

\section{Results}

2.1 The comparison between the imagine and estimate conditions

Participants reported a significantly higher emotion intensity and vividness of experience in the imagine condition compared to estimate condition (emotion intensity: $t(21)=3.69, p=.001$; vividness of experience: $t(21)=3.83, p=.001)$. The mean and SD of CFC 
EEG correlates of prospective cognition

scores (mean $\pm S D=42.41 \pm 4.90)$ in this study were similar to those reported in the initial paper of CFC by Strathman et al. (1994), showing that our participants' ability to consider their future fell in the normal range. The differences in EEG power and phase coherence between the imagine and estimate conditions were tested using two-way repeated measure ANOVAs for each frequency band respectively. There was no significant difference in EEG power between the two conditions (delta: $F(1,57)=1.72, p=.21$; theta: $F(1,57)=0.35, p$ $=.85$; alpha: $F(1,57)=3.48, p=.08$; beta: $F(1,57)=.14, p=.71)$. The imagine condition induced a significantly higher level of coherence across the DMN region pairs in the alpha band $(F(1,95)=4.28, p=.05)$ compared to the estimate condition. The differences of phase coherence in the delta, theta or beta bands failed to achieve statistical significance (delta: $F$ $(1,95)=1.72, p=.21 ;$ theta: $F(1,95)=0.09, p=.77$; beta: $F(1,95)=2.76, p=.11)$.

2.2 The comparisons of EEG correlates among the imagine, resting and waiting conditions

The 3 (Condition: imagine, resting and waiting) X 4 (Region: LMTG, MPFC, pC, RMTG) repeated measures ANOVA with power as the dependent variable showed significant main effects of condition and region, as well as a significant condition by region interaction across all frequency bands (delta: condition: $F(2,38)=31.07$, region: $F(3,57)=30.15$, interaction: $F(6,114)=31.07$; theta: condition: $F(2,38)=20.41$, region: $F(3,57)=15.14$, interaction: $F$ $(6,114)=23.66$; alpha: condition: $F(2,38)=20.08$, region: $F(3,57)=17.34$, interaction: $F(6$, $114)=25.44$; beta: condition: $F(2,38)=53.15$, region: $F(3,57)=18.11$, interaction: $F(6$, $114)=19.55$, all $p<.001$ ). Table 1 provides the statistical information of follow-up repeated measure ANOVAs between two conditions. In contrast to resting state, the imagine condition showed significantly lower power in the MPFC in all frequency bands. In addition, 
EEG correlates of prospective cognition

the imagine condition was associated with higher power in the bilateral MTG in the delta and theta bands, as well as higher power in the LMTG in the alpha band. As for the ANOVAs comparing the imagine and waiting conditions, there were significant main effects of region and condition, as well as significant condition by region interactions across all frequency bands. The imagine condition was associated with lower power within the MPFC in the alpha band compared to waiting. Moreover, the imagine condition was associated with higher power in MTG regions bilaterally across all frequency bands, as well as higher power in the $\mathrm{pC}$ in the delta band. Comparing resting and waiting conditions, there were significant main effects on region and condition, as well as significant condition by region interactions across all frequency bands. Resting was associated with higher power within the MPFC, LMTG and RMTG compared to waiting across all frequency bands. Figure 2 provides the EEG power of regional sources for each condition by frequency band.

In terms of phase coherence, the 3 (condition: imagine, resting and waiting) $\times 6$ (region pair: MPFC-LMTG, MPFC-pC, MPFC-RMTG, LMTG-pC, LMTG-RMTG, pC-RMTG) repeated measures ANOVAs showed significant main effects of condition and pathway across all frequency bands. The condition by pathway interaction was significant in the delta band only (delta: condition: $F(2,38)=8.81, p<.01$, region pair: $F(5,95)=28.24, p<.001$, interaction: $F(10,190)=3.89, p<.01$; theta: condition: $F(2,38)=8.29, p<.01$, region pair: $F$ $(5,95)=20.83, p<.001$, interaction: $F(10,190)=1.26, p=.29 ;$ alpha: condition: $F(2,38)=$ 7.18, $p<.01$, region pair: $F(5,95)=9.00, p<.001$, interaction: $F(10,190)=1.70, p=.14$; beta: condition: $F(2,38)=4.66, p=.02$, region pair: $F(5,95)=28.28, p<.001$, interaction: $F$ $(10,190)=1.88, p=.13)$. Table 2 provides the statistical information for post-hoc repeated measure ANOVAs between two conditions. Comparing the imagine with resting condition, 
EEG correlates of prospective cognition

there was a significant main effect of region pair across all frequency bands. The condition effect was significant in the delta, theta and alpha bands. Moreover, there was a significant condition by region pair interaction in the delta band, driven by higher phase coupling in the links of MPFC-pC, MPFC-RMTG, and LMTG-RMTG during the imagine condition. There was also a significant condition by pathway interaction in the theta band, driven by higher coupling among most region pairs during the imagine condition relative to resting, except for the link between MPFC and LMTG. While comparing the imagine with waiting condition, the main effect of pathway was significant across all frequency bands. The significant main effect of condition and the interaction were only seen in the delta band, which was driven by higher phase coupling between LMTG and RMTG during the imagine condition relative to waiting. In terms of the comparisons between resting and waiting, there was a significant main effect of region pair across frequency bands and a main effect of condition in the theta, alpha and beta bands. The interactions in the given frequency bands were non-significant. Figure 3 shows the PLV of each region pair across conditions by frequency band. Figure 4 marks the significant PLVs in the delta band in the imagine condition compared to resting and waiting states. 
EEG signatures of prospective cognition

\section{Discussion}

Previous research using $\mathrm{fMRI}$ has implicated the medial prefrontal cortex and medial temporal lobe within the DMN in episodic prospection. In this study we transformed the scalp recorded EEG from sensor level into brain source space to compare EEG band power and phase coherence during a prospection task and two control conditions: resting and waiting. There were a number of findings of note.

First, episodic prospection was associated with significant power reduction in the MPFC region. This was most marked for the comparison with rest where it was apparent across the four frequency bands examined. Results regarding the roles of MPFC in selfreferential processing have been inconsistent, with some studies showing elevated MPFC activity and some research reporting reduced MPFC activity during prospection. For instance, increased activity in the MPFC was demonstrated during prospective thinking (Addis, et al., 2007; Benoit, Gilbert, Frith, \& Burgess, 2012; Lavallee \& Persinger, 2010), but reduced activity in the MPFC was seen during an internally oriented but attention demanding task (Gilbert, Bird, Frith, \& Burgess, 2012). Furthermore, dorsal MPFC activity was increased during a self-referential judgment task while the ventral MPFC was attenuated, suggesting the multifaceted contribution of MPFC to internal oriented cognition (Gusnard, Akbudak, Shulman, \& Raichle, 2001). The inconsistency in a way reflects the fact that prospection is self-referential, as well as goal-directed and effortful. On the one hand, the attenuation of MPFC activity during episodic prospection compared to rest might suggest the reduced engagement of self-referential processes (Knyazev, 2013), which is unlikely to be the case in the current experiment. A more likely explanation for the current results is that prospection compared to rest requires more mental effort. Some of this inconsistency between studies 
EEG signatures of prospective cognition

with regard to MPFC therefore can be explained by the choice of different control conditions. For instance, Benoit et al. (2011) compared BOLD signals during the imagine and estimate conditions (both goal-directed and effortful tasks) and found reduced MPFC activity in the later, whereas in the current study we contrasted the imagine condition with pure rest. By including the resting state as a comparison in the current experiment we were able to isolate the neural correlates of the goal-directed nature of prospection.

Second, prospection was associated with increased power in the MTG. The effects were strongest for the comparison with waiting where they were present bilaterally for all frequency bands examined while for the comparison with rest they were only seen bilaterally in the delta and theta bands and in the left MTG in the alpha band. This finding was in line with previous $\mathrm{fMRI}$ studies which have implicated the DMN temporal regions in prospection - suggesting that envisioning the future involves the integration of functions subserved by temporal regions (i.e., autographical memory). The different direction of activity changes vis-a-vis the MPFC during prospection (increase rather than decrease) highlights the functional heterogeneity across different DMN regions (Laird, et al., 2009). Moreover, the fact that the difference was largest for prospection compared to waiting suggests that in the latter condition, once the decision to wait is made, little more active envisioning of the future occurs. In fact there is evidence to suggest that focusing on future outcomes while waiting makes the task more frustrating and difficult (Broyd et al., 2012). In contrast, during resting it is quite possible that there was some element of day dreaming occurring which may activate brain regions involving in both retrospective and prospective cognition. 
EEG signatures of prospective cognition

Third, compared to rest, prospection was associated with increased phase synchrony within the DMN regions particularly implicating temporal regions. The synchrony between the MPFC and $\mathrm{pC}$ was also enhanced in prospection compared to rest. This finding highlights the importance of taking a systems neuroscience view of the role of DMN in self-referential processes (Cortese et al., 2012). Research on functional connectivity is in its infancy and has been principally investigated using fMRI. Travis and colleagues recently uncovered evidence of frontal inter-hemispheric EEG coherence during meditation compared to rest, with sources overlapping the DMN (Travis et al., 2010). The work of Travis et al. provided promising evidence for using EEG coherence as a measure of spontaneous cognition. However, their study investigated the synchrony of EEG signals between electrode pairs on the scalp level rather than the source level. The source coherence is a more ideal way of measuring neural synchrony in EEG signals (Schoffelen \& Gross, 2009). To our knowledge, this study is the first to provide direct assessment of EEG source coherence between regions corresponding to the DMN. Further work is required to replicate and validate the current findings.

The way of the interaction between MPFC and MTG to provide the neural foundations for prospection is currently not well understood. The MTG sub-serves episodic memory and is active when an individual is consciously recollecting personal experience (Dickerson \& Eichenbaum, 2010). It coordinates associative processing of memory systems to provide the foundation for imagination (Bar, Aminoff, Mason, \& Fenske, 2007). It interacts with the MPFC, the area associated with self-projection and decision making, to mediate more complex processes such as perspective taking (Van Hoeck, et al., 2013) and mentalizing (Frith \& Frith, 2006). Fink and colleagues (2009) investigated the EEG activity on creative and 
EEG signatures of prospective cognition

habitual tasks (Fink, Graif, \& Neubauer, 2009). They reported that creative tasks enhanced alpha activity in the frontal, frontocentral and centrotemporal brain regions. The authors argued that although both creative and prospective thinking processes involved seeking associative information from episodic memory, creative thinking laid a greater stress on generating novel ideas, which possibly enhanced the activity in frontal area. By contrast, prospective thinking involves the anticipation of similar events and the elaboration of detail, which mainly activate the temporal lobe memory circuits.

It is noteworthy that this study found the most prominent effects related phase synchrony during the imagine condition in the delta band. The putative function of delta activity deserves further study. Delta oscillations are typically found in young children and during slow-wave sleep in adults (Anderson \& Horne, 2003). Recent evidence converges on showing that delta oscillations are involved in motivational processes and salience detection, with sources localized to medial frontal cortical structures, the nucleus accumbens and ventral tegmental area, overlapping with regions of the brain reward system (see Knyazev, 2007; Knyazev, 2012 for a detailed review). Given that the prospection conditions in the current study explicitly implicated incentives and future economic activity, the increase of delta oscillations is not be surprising. Indeed, delta oscillations are found to be enhanced in motivationally relevant situations when organisms have a need for reinforcements or rewards, such as hunger (Tallroth, Lindgren, Stenberg, Rosen, \& Agardh, 1990), sexual arousal (Schutter \& van Honk, 2004) and drug craving in addicts (Reid, Flammino, Howard, Nilsen, \& Prichep, 2006). Moreover, Bhattacharya and Petsche (2002) found increased delta synchrony in artists as compared with non-artists during imagery of paintings, suggesting the involvement of long-term memory may play a role in enhancing delta synchrony. 
EEG signatures of prospective cognition

We found few differences between the imagine and estimate conditions. There was however, found significantly higher PLVs in the alpha band in conjunction with greater selfratings of emotion intensity and vividness of experience. Given the fact that alpha activity is associative with restfulness and self-referential processes (Knyazev, 2013; Knyazev, et al., 2012) - processes that recruit the DMN (Andrews-Hanna, Smallwood, \& Spreng, 2014; Buckner \& Carroll, 2007), our participants appeared to engage in prospective thinking to a certain degree. In contrast to earlier studies (Klimesch, et al., 1994), there was no statistical significance in EEG power between the imagine and estimate conditions in this study. Using fMRI, Benoit et al. (2011) demonstrated that the imagine condition increased BOLD signals at DMN structures including the medial rostral prefrontal cortex, precuneus, bilateral parahippocampal gyri and retrosplenial corties compared to the estimate condition. This difference was explained in terms of the imagine condition involving a greater degree selfreferential processing as compared to the estimate condition. The finding of current study suggests that phase coherence in the alpha band may be a more sensitive measure of selfreferential processing than EEG power. In line with this argument, a simultaneous EEG-fMRI study by Jann et al. (2009) has demonstrated that BOLD correlates of EEG phase synchronization in the alpha band were located in DMN brain regions. To date most simultaneous EEG-fMRI research has focused on the correlation between EEG power and BOLD signals. It will be important to investigate the relation between phase coherence and DMN BOLD signals comprehensively. Moreover, the lack of difference in EEG power between the imagine and estimate conditions in this study may have another explanation. The reward magnitude and delay periods of the current prospection task were smaller than the study of Benoit (2011). Brain activation during personal prospective thinking appears to be modulated by features such as reward magnitude, plan detail, feasibility and temporal 
EEG signatures of prospective cognition

distance (Benoit, et al., 2011; Spreng, Gerlach, Turner, \& Schacter, 2015). Reduced amount of reward and the length of delay may diminish the effect of prospection on EEG power.

This study had several limitations that need to be considered when interpreting the results. First, the resting state EEG coherence in the delta band was higher in regions within rather than outside of the $\mathrm{DMN}$, but there was no significant difference in the theta, alpha and beta bands. This study provided preliminary evidence of the putative functional significance of resting EEG signals localized to the DMN. While the current finding is promising, a comprehensive examination of EEG network organization is required. Second, the DMN activity is characterized as very low frequency brain oscillations, normally less than $0.1 \mathrm{~Hz}$ (Broyd et al., 2009; Raichle, et al., 2001), which is slower than the delta band. Replication of the current finding and extending the examination of source coherence to very low frequency EEG are essential. Third, EEG regional sources reflected a collection of neuronal activity within an extended area instead of at a specific neuroanatomic coordinate. The activity of a seed region may involve signals from neighbouring structures. Further work using a whole brain analysis and/or co-registration of structural imaging data is recommended. Fourth, both EEG coherence and phase locking value may be contaminated by the smearing effect due to volume conduction. Although this study assessed neuronal interactions on the level of reconstructed sources, which diminished artefacts from volume conduction as compared to the neuronal interactions at the surface sensor level (Schoffelen \& Gross, 2009), the problem of volume conduction cannot be completely ruled out. Fifth, this study included a five minutes period of waiting to induce the feeling of delay. We recognise that participants may revert to a resting state during this condition at some points. However, prior research using the same condition showed a different pattern of mental 
EEG signatures of prospective cognition

activity during five minutes of waiting and five minutes of resting (Hsu, et al., 2015; Hsu, et al., 2013). Future research should include an online measure of participants' perceived mental state during waiting to clarify this issue. Finally, the findings of this study were based on a modest sample size. It is important for the future work to examine EEG signatures during prospection with a larger population including a group of clinical subjects.

\section{Experimental Procedure}

The study was approved by the School of Psychology Ethics Committee, University of Southampton.

\subsection{Participants}

Twenty-two students (18-35 years) recruited from the University of Southampton completed the study. The exclusion criteria were: (i) a history of brain injury; (ii) a diagnosis of neuropsychiatric disorder; (iii) use of any psychotropic substance more than twice in the last six months; and (iv) problems of substance abuse or dependence. All participants refrained from consuming caffeine or nicotine at least two hours prior to testing.

\subsection{Procedure}

Participants were familiarized with the electrophysiology laboratory and EEG recording procedures before informed consent was taken. They completed EEG recording during three conditions including prospection, resting and waiting. They also undertook a simple reaction 
EEG signatures of prospective cognition

time task although the data of this task were not presented here. The four conditions were counterbalanced using a Latin square table (Bailey, 2008). Consideration of Future

Consequences questionnaire (CFC; Strathman, et al., 1994) was used to evaluate participants' ability of considering time-distant versus immediate consequences of potential behaviours. The experiment took approximately $120 \mathrm{~min}$.

\subsubsection{Experimental paradigm}

The Prospection task was adapted from the protocol of Benoit, Gilbert and Burgess (2011). Participants were asked to imagine themselves spending money in a hypothetic scenario. The imagine condition required participants to concentrate on what they would see, smell, hear, and on the thoughts and feelings that they may experience in the specific scenario. For example, in the 'lunch, $f 20$ ' scenario participants were asked to elaborate as much detail as possible about where they would be and what might happen if they were given $\mathrm{f} 20$ to spend for a lunch event. There was also an estimate condition. In the estimate condition participants were asked to estimate the values of items that could purchase in these future scenarios. For example, participants estimated food cost for different items and thought about different combinations that could be purchased for lunch by $f 20$ in the lunch scenario. Prior to commencement participants were familiarised with a list of 24 scenarios (e.g. lunch, pub, and cinema) and a list of possible items that might be involved in the each of those episodes. Participants read through each item and briefly imagined whether they would purchase the item in the respective scenario and estimated the cost of each item. Participants were encouraged to think of other goods or items that they may purchase in the scenario and add them to the list. Finally participants rated the 24 everyday 
EEG signatures of prospective cognition

scenarios in terms of expected level of enjoyment. The twenty scenarios with the highest ratings were selected for the main task. The four scenarios not chosen were employed as practice. Each scenario included an imagine and an estimate condition. Each scenario was randomly assigned one of the combinations of five reward magnitudes $(£ 10,12,15,18,20)$ and four delays (30,60, 90, 120 days). The imagine and estimate conditions of a specific scenario were assigned to the same combination of reward magnitude and delay period. The resulting 40 trials were presented in a random order, which were split into two blocks with a short break in the middle of the task. The imagining or estimating duration for each trial lasted for 30 seconds. Following each scenario participants answered two self-rating questions about emotional intensity and vividness of experience on a four point scale. The first question was "How do you feel while imagining the episode?" (for the imagine condition) or "How do you feel while thinking about what the money could purchase?" (for the estimate condition; $1=$ detachment; 2 =mild; $3=$ moderate; $4=$ highly emotional). The second question was: "Please rate the degree to which you had the feeling of mentally experiencing the event" (1=none; $2=$ mild; $3=$ moderate; $4=$ highly experienced).

In the resting condition, participants were asked to relax for five minutes and focus on the fixation cross on the monitor. In the waiting condition they were given a choice to wait for five minutes to win a ticket for a $f 20$ lottery draw or to immediately terminate the waiting period. All participants agreed to wait.

\subsection{EEG data acquisition}


EEG signatures of prospective cognition

EEG was recorded using a Neuroscan Synamps 70 channel system via 24 bit A/D converter with direct current procedure, combined with a $70 \mathrm{~Hz}$ low-pass filter, sampling at $500 \mathrm{~Hz}$ and referenced to an electrode on the nose. Participants were fitted with an electrode cap with 66 equidistant $\mathrm{Ag} / \mathrm{AgCl}$ electrodes (Easycap; Hersching, Germany). EEG data from the first 55 electrodes were used for analysis (Figure 1). We discarded the other 11 electrodes located at the outmost circle on the cap because previous studies have shown these electrodes were frequently contaminated with artefacts. Impedance was kept below 5 $\mathrm{k} \Omega$. Electro-oculogram (EOG) was record using $\mathrm{Ag} / \mathrm{AgCl}$ electrodes placed below the left and right eyes to measure vertical movements.

\subsection{EEG data processing}

EEG data processing was performed using Brain Electrical Source Analysis program (BESA research 6.0; www.besa.de). BESA was developed by Michael Scherg and colleagues on the basis of 30 years of experience in human brain research for localizing brain sources equivalent dipoles (Scherg \& Picton, 1991). The implementation of BESA source estimation has been tested using simulated data, showing a reasonable degree of accuracy with an average localization error of $1.4 \mathrm{~cm}$ (Miltner, Braun, Johnson, Simpson, \& Ruchkin, 1994).

BESA is widely used for source analyses in EEG and MEG research. In this study we performed source analysis on the basis of a previous identified BESA source model reported in the resting-state magnetoencephalographic (MEG) literature (Franzen et al., 2013; Wilson et al., 2013). The MEG source model contains 29 nodes over the whole brain fitted to individual's structural image following coregistration (Wilson, et al., 2013). The coordinates 
EEG signatures of prospective cognition

of the 29 nodes were used as the initial starting locations for the spatiotemporal dipole modeling of EEG data. Standard source montage was then generated consisting of 29 nodes with triple orthogonal dipoles based on the coordinators (Scherg, Ille, Bornfleth, \& Berg, 2002). The source montage was used to estimate regional neural activity of the resting network. We first performed artefact rejection for the EEG scalp recorded data based on a fixed threshold method supplemented with careful visual inspection. Then the artefactcleaned data were transformed into the source montage via inverse spatial filtering (Scherg \& Berg, 1996; Scherg \& Picton, 1991). Detailed description of spatial filtering could be found in relevant publications (e.g. Franzen, et al., 2013). Briefly, it involves creating a linear inverse operator of the lead field matrix $\left(L=\left(l_{1}, I_{2}, \ldots, I_{n}\right)\right)$, which contains the lead field vector of each source orientation in the model. Scalp recorded EEG signals can be represented by the linear overlap coming from the source regions, i.e. $U=L \times S+$ noise; $U$ states the EEG scalp recorded signals in time series, with each source region $\left(\mathrm{S}_{\mathrm{i}}\right.$, row $\mathrm{i}$ in source matrix $\left.\mathrm{S}\right)$ having an unknown level of activity over time. Then regional source activity can be reconstructed by inverting matrix $L$ (i.e., $S=L^{-1} \times U-L^{-1} \times$ noise), with $L^{-1}$ being a spatial filter that reconstructs the magnitude of each individual source in brain area $S_{i}$ over time while suppressing activity from all other brain areas.

After being transformed into the source montage, the signals were divided into epochs of $1600 \mathrm{~ms}$. For each condition and participant, the epochs were transformed into timefrequency space using complex demodulation (Hoechstetter et al., 2004; Papp \& Ktonas, 1977). The EEG power of each region, as well as the coherence and phase locking value of each region pair were calculated with frequency sampling between 1 and $34 \mathrm{~Hz}$ in steps of $0.5 \mathrm{~Hz}$ and $100 \mathrm{~ms}$ at the epochs. 
EEG signatures of prospective cognition

This study focused on four DMN seed regions, including the midline anterior and posterior hubs, i.e. medial prefrontal gyrus (MPFC) and precuneus $(\mathrm{pC})$, and areas related to episode memory, i.e. left and right middle temporal gyrus (LMTG \& RMTG). To assess if the chosen seeds formed a network, we compared the degree of coherence between $\mathrm{pC}$ and three DMN nodes and the coherence between $\mathrm{pC}$ and three randomly selected nodes outside of the network for each frequency band respectively. We compared the harmonic means of coherence within and outside of the DMN to denote the average of coherence rates and to reduce the influence of deviant values. The difference of coherence within and outside of the DMN was assessed using paired t test for each frequency band separately. The results showed the coherences within the DMN was significantly higher than those outside of the DMN in the delta band $\left(\mathrm{H}_{\text {within }}=.015, \mathrm{H}_{\text {outside }}=.012, t(19)=2.99, p=.008\right)$. There was no significant difference of networks in the other frequency bands (theta: $\mathrm{H}_{\text {within }}$ $=.013, \mathrm{H}_{\text {outside }}=.012, t(19)=.46, p=.65 ;$ alpha: $\mathrm{H}_{\text {within }}=.018, \mathrm{H}_{\text {outside }}=.015, t(19)=1.05, p$ $=.31$; beta: $\left.\mathrm{H}_{\text {within }}=.012, \mathrm{H}_{\text {outside }}=.014, t(19)=-1.36, p=.19\right)$.

Next, the EEG power within each of the four seed regions was extracted and natural log transformed to normalize the distribution (Gasser, Bächer, \& Möcks, 1982). Phase coherence was computed using phase-locking value (PLV) through the method described by Lachaux, Rodriguez, Martinerie and Varela (1999). We adapted PLV as the coherence measure because it was independent of the effects of amplitude or power, reflecting the integration between two spatially apart signals based on the correlation of two normalized spectral density functions. The range of PLV was from 0 to 1 . The higher the PLV value indicated the higher synchrony between two signals at a given frequency. For each participant PLVs were calculated over epochs for each region pair by frequency band and 
EEG signatures of prospective cognition

condition. We examined the EEG power and PLVs for each condition in four frequency bands: delta $(\Delta)$ : 1-4 Hz; theta $(\Theta): 4-8 \mathrm{~Hz}$; alpha $(\alpha): 8-12 \mathrm{~Hz}$; and beta $(\beta): 12-34 \mathrm{~Hz}$.

\subsection{Data analysis}

Two participants were excluded from data analysis. One of them did not complete the waiting condition because of a technical problem. The other participant's EEG quality was poor, probably due to her afro hairstyle. The data of the remaining 20 participants were used for analysis.

We first examined if the experimental manipulation of prospection was successful. Paired $t$ test was used to test the difference of self-rating emotional intensity and vividness of experience between the imagine and estimate conditions. The differences of EEG power and phase coherence between imagine and estimate conditions were tested using a 2 (Condition: imagine vs. estimate) X 4 (Region: LMTG, MPFC, pC, RMTG) and a 2 X 6 (Region pair: MPFC-LMTG, MPFC-pC, MPFC-RMTG, LMTG-pC, LMTG-RMTG, pC-RMTG) repeated measure ANOVA.

Subsequently we tested for differences of power and phase coherence using a number of two-way repeated measure ANOVAs with condition (imagine, resting and waiting) and region (i.e. 4 sources: LMTG, MPFC, pC, RMTG) or region pair (i.e. 6 pathways: MPFC-LMTG, MPFC-pC, MPFC-RMTG, LMTG-pC, LMTG-RMTG, pC-RMTG) as the within subject variables for each frequency band with power or phase coherence as the dependent variable. Because these ANOVAs indicated significant main effects of condition and region or region pair, as well as significant interactions, follow-up analyses were performed to test the 
EEG signatures of prospective cognition

difference between two conditions (imagine vs. rest, imagine vs. wait, and rest vs. wait) using 2X4 (for EEG power) or 2X6 (for phase coherence) repeated measures ANOVAs. Simple main effect analyses were applied to examine significant interactions with Bonferroni correction for multiple comparisons.

\section{Conclusion}

Here we extended previous research of neural mechanisms underpinning episodic prospection from fMRI to EEG. Compared to resting and waiting, episodic prospection was associated with significantly higher activity in the MPFC and lower activity in the MTGs across frequency bands and a high level of MPFC-MTG and bilateral MTGs phase synchrony in the delta band. These results highlight the different roles of DMN regions in selfreferential cognition and the importance of medial frontal and medial temporal gyrus in prospective cognition. 


\section{Financial Disclosures}

Chia-Fen Hsu reports no potential conflicts of interest. Dr. Sonuga-Barke has potential conflicts of interest in relation to Shire pharmaceuticals - speaker fees, consultancy, advisory board membership, research support and conference attendance funds. Janssen Cilag - speaker fees. Visiting chairs at Ghent University and Aarhus University. Grants awarded from MRC, ESRC, Wellcome Trust, Solent NHS Trust, European Union, Child Health Research Foundation New Zealand, NIHR, Nuffield Foundation, Fonds Wetenschappelijk Onderzoek - Vlaanderen (FWO).

\section{Acknowledgements}

The authors would like to thank Dr. Roland G. Benoit for sharing the prospection task, Dr. Tony W. Wilson for providing the source montage model and advices for data processing, Dr. Andre Waelkens for offering technical support of BESA software, and Dr. Yen Yu for providing valuable advice on time-frequency analysis. We also acknowledge the contribution of Nicholas Benikos to this study. This project was funded by a scholarship from the Ministry of Education, Taiwan to the first author. 
EEG signatures of prospective cognition

\section{Figure captions}

\section{Figure 1}

The regional source model. Scalp recorded EEG from 55 electrodes was fitted to the 29regional source model (Wilson, et al., 2013). Left: scalp location of the electrodes. Right: the source montage with 29 nodes. Each node was spaced equidistant apart $\left(>1 \mathrm{~cm}^{3}\right)$. Each source node represented activity over an extended brain region rather than the amount of activation at a specific neuroanatomical coordinate. For example, the arrow pointed to the MPFC seed region, which covered the medial and superior frontal gyrus areas.

\section{Figure 2}

EEG power (In) within each of the regional sources during the imagine (red), resting (blue) and waiting (green) conditions by frequency band. In contrast to resting condition, the imaging condition was associated with higher power in bilateral MTG in the delta and theta bands and left MTG in the alpha band, as well as lower power in the MPFC across all frequency bands. In contrast to waiting condition, the imagine condition was associated with higher power in bilateral MTG across all frequency bands and higher power in $\mathrm{pC}$ region in the delta band. Comparing resting and waiting conditions, the resting condition was associated with higher power in MPFC and bilateral MTG across all frequency bands. Asterisks indicated statistical significance revealed by analyses of simple effects for pairwise comparisons of conditions using Bonferroni correction. MPFC: medial prefrontal gyrus; pC: precuneus; LMTG: left middle temporal gyrus: RMTG: right middle temporal gyrus. Bars represent standard errors of the mean. 


\section{Figure 3}

Phase coherence: Phase locking values during the imagine (red), resting (blue) and waiting (green) conditions by frequency bands. The imaging condition was associated with higher delta phase coupling in the links of MPFC-pC, MPFC-RMTG, and LMTG-RMTG, as well as higher theta phase coupling in the links of MPFC - pC, MPFC- RMTG; pc - LMTG; LMTG RMTG, pc - RMTG compared to resting condition. Moreover, the imagine condition was associated with higher delta phase coupling in the links of LMTG - RMTG compared to waiting condition. Asterisks indicated statistical significance revealed by analyses of simple effects for pairwise comparisons of conditions using Bonferroni correction. MPFC: medial prefrontal gyrus; pC: precuneus; LMTG: left medial temporal gyrus; RMTG: right medial temporal gyrus (RMTG). Bars represent standard errors of the mean.

\section{Figure 4}

Differential patterns of phase coherence in the delta band. The phase coupling between LMTG and RMTG during the imagine condition was significantly higher compared to both

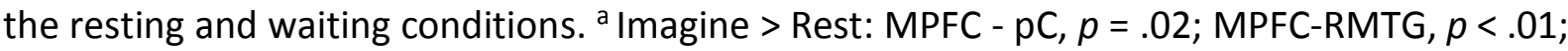
LMTG-RMTG, $p=.001 .{ }^{\mathrm{b}}$ Imagine $>$ Wait: LMTG-RMTG, $p<.01$. The simple effect analysis used Bonferroni correction. MPFC: medial prefrontal gyrus; pC: precuneus; LMTG: left medial temporal gyrus; RMTG: right medial temporal gyrus. 


\section{Reference}

Addis, D. R., Wong, A. T., \& Schacter, D. L. (2007). Remembering the past and imagining the future: Common and distinct neural substrates during event construction and elaboration. Neuropsychologia, 45(7), 1363-1377.

Anderson, C., \& Horne, J. A. (2003). Prefrontal cortex: Links between low frequency delta EEG in sleep and neuropsychological performance in healthy, older people. Psychophysiology, 40(3), 349-357.

Andrews-Hanna, J. R., Smallwood, J., \& Spreng, R. N. (2014). The default network and self-generated thought: component processes, dynamic control, and clinical relevance. Annals of the New York Academy of Sciences, 1316, 29-52.

Bailey, R. A. (2008). Row-column designs. Design of Comparative Experiments. (pp. 105-115). Cambridge: Cambridge University Press.

Bar, M. (2009). The proactive brain: Memory for predictions. Philosophical Transactions of the Royal Society of London. Series B, Biological Sciences, 364(1521), 1235-1243.

Bar, M., Aminoff, E., Mason, M., \& Fenske, M. (2007). The units of thought. Hippocampus, 17(6), 420-428.

Benoit, R. G., Gilbert, S. J., \& Burgess, P. W. (2011). A neural mechanism mediating the impact of episodic prospection on farsighted decisions. The Journal of Neuroscience, 31(18), 67716779.

Benoit, R. G., Gilbert, S. J., Frith, C. D., \& Burgess, P. W. (2012). Rostral prefrontal cortex and the focus of attention in prospective memory. Cerebral Cortex, 22(8), 1876-1886.

Boyer, P. (2008). Evolutionary economics of mental time travel? Trends in cognitive sciences, 12(6), 219-224.

Broyd, S. J., Demanuele, C., Debener, S., Helps, S. K., James, C. J., \& Sonuga-Barke, E. J. S. (2009). Default-mode brain dysfunction in mental disorders: A systematic review. Neuroscience and Biobehavioral Reviews, 33(3), 279-296.

Broyd, S. J., Richards, H. J., Helps, S. K., Chronaki, G., Bamford, S., \& Sonuga-Barke, E. J. S. (2012). Electrophysiological markers of the motivational salience of delay imposition and escape. Neuropsychologia, 50(5), 965-972.

Buckner, R. L., Andrews-Hanna, J. R., \& Schacter, D. L. (2008). The brain's default network: Anatomy, function, and relevance to disease. Annals of the New York Academy of Sciences, 1124, 1-38.

Buckner, R. L., \& Carroll, D. C. (2007). Self-projection and the brain. Trends in Cognitive Sciences, 11(2), 49-57.

Cannon, R., Kerson, C., \& Hampshire, A. (2011). sLORETA and fMRI detection of medial prefrontal default network anomalies in adult ADHD. Journal of Neurotherapy, 15(4), 358-373.

Cannon, R., Kerson, C., Hampshire, A., \& Garner, C. L. (2012). Pilot Data Assessing the Functional Integrity of the Default Network in Adult ADHD with fMRI and sLORETA. Journal of Neurotherapy, 16(4), 246-263.

Christoff, K. (2012). Undirected thought: Neural determinants and correlates. Brain Research, 1428, 51-59.

Christoff, K., Gordon, A. M., Smallwood, J., Smith, R., \& Schooler, J. W. (2009). Experience sampling during $f M R I$ reveals default network and executive system contributions to mind wandering. Proceedings of the National Academy of Sciences of the United States of America, 106(21), 8719-8724.

Cortese, S., Kelly, C., Chabernaud, C., Proal, E., Di Martino, A., Milham, M. P., et al. (2012). Toward systems neuroscience of ADHD: A meta-analysis of $55 \mathrm{fMRI}$ studies. American Journal of Psychiatry, 169(10), 1038-1055.

Dickerson, B. C., \& Eichenbaum, H. (2010). The episodic memory system: Neurocircuitry and disorders. Neuropsychopharmacology, 35(1), 86-104. 
Fink, A., Graif, B., \& Neubauer, A. C. (2009). Brain correlates underlying creative thinking: EEG alpha activity in professional vs. novice dancers. Neuroimage, 46(3), 854-862.

Fox, K. C., Spreng, R. N., Ellamil, M., Andrews-Hanna, J. R., \& Christoff, K. (2015). The wandering brain: Meta-analysis of functional neuroimaging studies of mind-wandering and related spontaneous thought processes. Neuroimage, 111, 611-621.

Franzen, J. D., Heinrichs-Graham, E., White, M. L., Wetzel, M. W., Knott, N. L., \& Wilson, T. W. (2013). Atypical coupling between posterior regions of the default mode network in AttentionDeficit/Hyperactivity Disorder: A pharmaco-magnetoencephalography study. Journal of Psychiatry and Neuroscience, 38(5), 333-340.

Frith, C. D., \& Frith, U. (2006). The neural basis of mentalizing. Neuron, 50(4), 531-534.

Gasser, T., Bächer, P., \& Möcks, J. (1982). Transformations towards the normal distribution of broad band spectral parameters of the EEG. Electroencephalography and Clinical Neurophysiology, 53(1), 119-124.

Gilbert, S. J., Bird, G., Frith, C. D., \& Burgess, P. W. (2012). Does "task difficulty" explain "task-induced deactivation?". Frontier in Psychology, 3, 125.

Gusnard, D. A., Akbudak, E., Shulman, G. L., \& Raichle, M. E. (2001). Medial prefrontal cortex and self-referential mental activity: Relation to a default mode of brain function. Proceedings of the National Academy of Sciences of the United States of America, 98(7), 4259-4264.

Helps, S. K., Broyd, S. J., James, C. J., Karl, A., Chen, W., \& Sonuga-Barke, E. J. S. (2010). Altered spontaneous low frequency brain activity in Attention Deficit/Hyperactivity Disorder. Brain Research, 1322, 132-143.

Helps, S. K., Broyd, S. J., James, C. J., Karl, A., \& Sonuga-Barke, E. S. (2009). The attenuation of very low frequency brain oscillations in transitions from a rest state to active attention. Journal of Psychophysiology, 23(4), 191-198.

Helps, S. K., James, C., Debener, S., Karl, A., \& Sonuga-Barke, E. J. S. (2008). Very low frequency EEG oscillations and the resting brain in young adults: A preliminary study of localisation, stability and association with symptoms of inattention. Journal of Neural Transmission, 115(2), 279285.

Hiltunen, T., Kantola, J., Abou Elseoud, A., Lepola, P., Suominen, K., Starck, T., et al. (2014). Infra-slow EEG fluctuations are correlated with resting-state network dynamics in FMRI. Journal of Neuroscience, 34(2), 356-362.

Hoechstetter, K., Bornfleth, H., Weckesser, D., Ille, N., Berg, P., \& Scherg, M. (2004). BESA source coherence: a new method to study cortical oscillatory coupling. Brain Topography, 16(4), 233-238.

Hsu, C.-F., Benikos, N., \& Sonuga-Barke, E. J. S. (2015). Spontaneous activity in the waiting brain: A marker of impulsive choice in attention-deficit/hyperactivity disorder? Developmental Cognitive Neuroscience, 12, 114-122.

Hsu, C.-F., Broyd, S. J., Helps, S. K., Benikos, N., \& Sonuga-Barke, E. J. S. (2013). “Can waiting awaken the resting brain?" A comparison of waiting- and cognitive task-induced attenuation of very low frequency neural oscillations. Brain Research, 1524, 34-43.

Jann, K., Dierks, T., Boesch, C., Kottlow, M., Strik, W., \& Koenig, T. (2009). BOLD correlates of EEG alpha phase-locking and the fMRI default mode network. Neuroimage, 45(3), 903-916.

Kim, H. (2012). A dual-subsystem model of the brain's default network: Self-referential processing, memory retrieval processes, and autobiographical memory retrieval. Neuroimage, 61(4), 966-977.

Klimesch, W. (1999). EEG alpha and theta oscillations reflect cognitive and memory performance: a review and analysis. Brain Research Reviews, 29(2-3), 169-195.

Klimesch, W., Doppelmayr, M., Pachinger, T., \& Ripper, B. (1997). Brain oscillations and human memory: EEG correlates in the upper alpha and theta band. Neuroscience Letters, 238(1-2), 9-12. 
Klimesch, W., Schimke, H., \& Schwaiger, J. (1994). Episodic and semantic memory: an analysis in the EEG theta and alpha band. Electroencephalography and Clinical Neurophysiology, 91(6), 428441.

Knyazev, G. G. (2007). Motivation, emotion, and their inhibitory control mirrored in brain oscillations. Neuroscience and Biobehavioral Reviews, 31(3), 377-395.

Knyazev, G. G. (2012). EEG delta oscillations as a correlate of basic homeostatic and motivational processes. Neuroscience and Biobehavioral Reviews, 36(1), 677-695.

Knyazev, G. G. (2013). EEG correlates of self-referential processing. Frontiers in Human Neuroscience, 7, 264.

Knyazev, G. G., Savostyanov, A. N., Volf, N. V., Liou, M., \& Bocharov, A. V. (2012). EEG correlates of spontaneous self-referential thoughts: A cross-cultural study. International Journal of Psychophysiology, 86(2), 173-181.

Kwan, D., Craver, C. F., Green, L., Myerson, J., Boyer, P., \& Rosenbaum, R. S. (2012). Future decisionmaking without episodic mental time travel. Hippocampus, 22(6), 1215-1219.

Kwan, D., Craver, C. F., Green, L., Myerson, J., Gao, F., Black, S. E., et al. (2015). Cueing the personal future to reduce discounting in intertemporal choice: Is episodic prospection necessary? Hippocampus, 25(4), 432-443.

Lachaux, J. P., Rodriguez, E., Martinerie, J., \& Varela, F. J. (1999). Measuring phase synchrony in brain signals. Human Brain Mapping, 8(4), 194-208.

Laird, A. R., Eickhoff, S. B., Li, K., Robin, D. A., Glahn, D. C., \& Fox, P. T. (2009). Investigating the functional heterogeneity of the default mode network using coordinate-based meta-analytic modeling. Journal of Neuroscience, 29(46), 14496-14505.

Laufs, H., Krakow, K., Sterzer, P., Eger, E., Beyerle, A., Salek-Haddadi, A., et al. (2003). Electroencephalographic signatures of attentional and cognitive default modes in spontaneous brain activity fluctuations at rest. Proceedings of the National Academy of Sciences of the United States of America, 100(19), 11053-11058.

Lavallee, C. F., \& Persinger, M. A. (2010). A LORETA study of mental time travel: Similar and distinct electrophysiological correlates of re-experiencing past events and pre-experiencing future events. Consciousness and Cognition, 19(4), 1037-1044.

Li, W., Mai, X., \& Liu, C. (2014). The Default Mode Network and Social Understanding of Others: What do Brain Connectivity Studies Tell Us. Frontiers in Human Neuroscience, 8, 74.

Lin, H., \& Epstein, L. H. (2014). Living in the moment: Effects of time perspective and emotional valence of episodic thinking on delay discounting. Behavioral Neuroscience, 128(1), 12-19.

Mantini, D., Perrucci, M. G., Gratta, C. D., Romani, G. L., \& Corbetta, M. (2007). Electrophysiological signatures of resting state networks in the human brain. Proceedings of the National Academy of Sciences of the United States of America, 104(32), 13170-13175.

Meltzer, J. A., Negishi, M., Mayes, L. C., \& Constable, R. T. (2007). Individual differences in EEG theta and alpha dynamics during working memory correlate with $\mathrm{fMRI}$ responses across subjects. Clinical Neurophysiology, 118(11), 2419-2436.

Miltner, W., Braun, C., Johnson, R., Jr., Simpson, G. V., \& Ruchkin, D. S. (1994). A test of brain electrical source analysis (BESA): a simulation study. Electroencephalography Clinical Neurophysiology, 91(4), 295-310.

Papp, N., \& Ktonas, P. (1977). Critical evaluation of complex demodulation techniques for the quantification of bioelectrical activity. Biomedical Sciences Instrumentation, 13, 135-145.

Peters, J., \& Büchel, C. (2010). Episodic future thinking reduces reward delay discounting through an enhancement of prefrontal-mediotemporal interactions. Neuron, 66(1), 138-148.

Raichle, M. E., MacLeod, A. M., Snyder, A. Z., Powers, W. J., Gusnard, D. A., \& Gordon, L. S. (2001). A default mode of brain function. Proceedings of the National Academy of Sciences of the United States of America, 98(2), 676-682. 
Reid, M. S., Flammino, F., Howard, B., Nilsen, D., \& Prichep, L. S. (2006). Topographic imaging of quantitative EEG in response to smoked cocaine self-administration in humans. Neuropsychopharmacology, 31(4), 872-884.

Reniers, R. L. E. P., Corcoran, R., Völlm, B. A., Mashru, A., Howard, R., \& Liddle, P. F. (2012). Moral decision-making, ToM, empathy and the default mode network. Biological Psychology, 90(3), 202-210.

Scheeringa, R., Petersson, K. M., Oostenveld, R., Norris, D. G., Hagoort, P., \& Bastiaansen, M. C. (2009). Trial-by-trial coupling between EEG and BOLD identifies networks related to alpha and theta EEG power increases during working memory maintenance. Neuroimage, 44(3), 1224-1238.

Scherg, M., \& Berg, P. (1996). New concepts of brain source imaging and localization. Electroencephalography and Clinical Neurophysiology. Supplement, 46, 127-137.

Scherg, M., Ille, N., Bornfleth, H., \& Berg, P. (2002). Advanced tools for digital EEG review: virtual source montages, whole-head mapping, correlation, and phase analysis. Journal of Clinical Neurophysiology, 19(2), 91-112.

Scherg, M., \& Picton, T. W. (1991). Separation and identification of event-related potential components by brain electric source analysis. Electroencephalography and Clinical Neurophysiology. Supplement, 42, 24-37.

Schoffelen, J.-M., \& Gross, J. (2009). Source connectivity analysis with MEG and EEG. Human Brain Mapping, 30(6), 1857-1865.

Schutter, D. J., \& van Honk, J. (2004). Decoupling of midfrontal delta-beta oscillations after testosterone administration. International Journal of Psychophysiology, 53(1), 71-73.

Smallwood, J., Brown, K., Baird, B., \& Schooler, J. W. (2012). Cooperation between the default mode network and the frontal-parietal network in the production of an internal train of thought. Brain Research, 1428, 60-70.

Sonuga-Barke, E. J. S., \& Fairchild, G. (2012). Neuroeconomics of Attention-Deficit/Hyperactivity Disorder: Differential influences of medial, dorsal, and ventral prefrontal brain networks on suboptimal decision making? Biological Psychiatry, 72(2), 126-133.

Spreng, R. N., Gerlach, K. D., Turner, G. R., \& Schacter, D. L. (2015). Autobiographical Planning and the Brain: Activation and Its Modulation by Qualitative Features. Journal of Cognitive Neuroscience, 1-11.

Spreng, R. N., \& Grady, C. L. (2010). Patterns of brain activity supporting autobiographical memory, prospection, and theory of mind, and their relationship to the default mode network. Journal of Cognitive Neuroscience, 22(6), 1112-1123.

Spreng, R. N., Mar, R. A., \& Kim, A. S. (2009). The common neural basis of autobiographical memory, prospection, navigation, theory of mind, and the default mode: a quantitative meta-analysis. Journal Cognitive Neuroscience, 21(3), 489-510.

Strathman, A., Gleicher, F., Boninger, D. S., \& Edwards, C. S. (1994). The Consideration of Future Consequences - Weighing Immediate and Distant Outcomes of Behavior. Journal of Personality and Social Psychology, 66(4), 742-752.

Szpunar, K. K., Watson, J. M., \& McDermott, K. B. (2007). Neural substrates of envisioning the future. Proceedings of the National Academy of Sciences, 104(2), 642-647.

Tallroth, G., Lindgren, M., Stenberg, G., Rosen, I., \& Agardh, C. D. (1990). Neurophysiological changes during insulin-induced hypoglycaemia and in the recovery period following glucose infusion in type 1 (insulin-dependent) diabetes mellitus and in normal man. Diabetologia, 33(5), 319323.

Travis, F., Haaga, D. A. F., Hagelin, J., Tanner, M., Arenander, A., Nidich, S., et al. (2010). A selfreferential default brain state: Patterns of coherence, power, and eLORETA sources during eyes-closed rest and transcendental meditation practice. Cognitive Processing, 11(1), 21-30.

Tulving, E. (2002). Episodic memory: From mind to brain. Annual Review of Psychology, 53(1), 1-25. 
EEG signatures of prospective cognition

Van Hoeck, N., Ma, N., Ampe, L., Baetens, K., Vandekerckhove, M., \& Van Overwalle, F. (2013). Counterfactual thinking: An fMRI study on changing the past for a better future. Social Cognitive and Affective Neuroscience, 8(5), 556-564.

Wilson, T. W., Franzen, J. D., Heinrichs-Graham, E., White, M. L., Knott, N. L., \& Wetzel, M. W. (2013). Broadband neurophysiological abnormalities in the medial prefrontal region of the defaultmode network in adults with ADHD. Human Brain Mapping, 34(3), 566-574. 